\title{
EDITORIAL
}

\section{Exposure science: A need to focus on conducting scientific studies, rather than debating its concepts}

\author{
Journal of Exposure Science and Environmental Epidemiology (2013) \\ 23, 455-456; doi:10.1038/jes.2013.43
}

Over the past $25+$ years, Exposure Science has provided a wealth of credible experimental, field and theoretical-based research, including the how, where and when elevated exposures occur, and ultimately how to mitigate or prevent contact with chemical contaminants and other stressors. It is therefore puzzling that, over the past 7 years, many Exposure Scientists have not taken advantage of our successes, but instead have focused on the adequacy of its Scientific Concepts and Terminology. This has tended to distract us from addressing the acute need for resources to conduct state-of-the-art scientific studies to solve problems. I believe that one unintended consequence of the distraction has been the lack of drive for sustained funding of exposure science research.

There has, however, recently been one well-appreciated forward movement, that is, the increased funding of research in exposure biology. Unfortunately, it has been insufficient in meeting the need for adequate funding to sustain the field as a whole, and US scientists have failed to effectively promote other areas of exposure science. For example, they have not provided support for the cogent arguments made by ISES over 4 years ago to counteract the impact of Congress's continued blockage of EPA's external/internal funding of observational studies of children's exposures. Observational research in natural settings has long been a cornerstone of the field's ability to address environmental health problems, and the lack of such studies has allowed the proliferation of mosquito misters, analogous to water sprinklers, into residential neighborhoods, exposing both children and wildlife to this new "broadcast" method of delivering pesticides. The continued decline in the role of Exposure Science in the conduct of public health research in the United States is now evident in the National Children's Study (NCS). The NCS is rethinking its comprehensive study design, and moving away from hypotheses testing. Hypothesis testing studies are required to understand how toxic exposures affect children in their homes and neighborhood environments, which was a main reason for the initial funding of the program.

A unifying concept, developed to overcome some of the confusion between exposure science and technology for biological specimen analysis, is the "exposome." It is a very interesting idea, as defined by Wild" to "encompass the life-course environmental exposures (including lifestyle factors) from the prenatal period onward to death. Unfortunately, the exposome concept has not been unifying. Rather, it has been divisive because of the way it has been interpreted by some members of the environmental health community. Tension developed between those who favor reliance on only one of the two essential components of exposure science: external and internal markers of exposure, rather than recognizing that they are complementary. ${ }^{2}$ Internal markers of exposure have been effective tools for recognizing that exposure has occurred. For example, levels in biological fluids have been effectively used in the NHANES to establish baseline distributions of many chemicals that can be found in blood and urine; and biomarkers referred to as Biological Exposure Indices (BEls) have been increasingly used in occupational settings to establish worker exposures since the 1980s. These uses predate the idea of the "exposome" and are important measurements in the exposure science "toolbox". The energy associated with the promotion of the exposome concept, however, has been the interpreted, by some, to promote internal markers of exposure as the best, or as the only, way to employ the principles of exposure science. To date, many internal markers of exposure have only been able to define that "you have been exposed" and not how, when, where, or to how much.

Some internal markers have been more successful in improving hazard assessment for risk assessment, but we need to recognize they provide only one part of the information needed to appropriately complete a risk assessment-the other part, for those who do not "get it," is quantitative exposure assessment. Further, the current suite of "omic" tools that have been touted as the next wave of internal markers of exposure appear to have little to offer in the way of characterizing the quantitative range of data required to help better understand whether or not a person or a group of people have been significantly exposed to a toxic agent. We need to design and complete validation studies that address the quantitative use of these new markers to establish the intensity, duration, and variability of exposure. The lack of specificity for many internal markers is a major reason for the continuing consternation with the "exposome" by professionals in the field who understand the serious need for measuring external markers of exposure.

Historically, and as stated above, external markers of exposures have provided a cornerstone of exposure science both in occupational and environmental situations. Because of the nature of such measurements, they can be minimally invasive, for example, requiring the wearing personal monitors or ingesting a duplicate meal. Sometimes the studies have been limited in scope, dealing with small picture issues, and confined to an individual or a population sub-group. However, as demonstrated in TEAM, NHEXAS, and EXPOLIS and other large and complex studies, they can be planned and implemented to provide population-based information on the magnitude and intra-population variability of exposure. Each of these larger studies examined exposures of individuals within a population, in many cases using both external and internal marker measurements, in their "natural environments" with the ultimate goal of mitigating exposures that were potentially harmful to human health.

In 2011, Rappaport ${ }^{3}$ and I published an editorial in which we attempted to outline the problems that have arisen with the interpretation of the exposome over recent years. We focused on the idea that internal markers of exposure are beneficial for characterizing exposure-disease response relationships. The field of exposure has already had a major role in providing information about what led to the exposures that could cause an adverse health outcome, and how they can be mitigated. A 2012 article by Wild ${ }^{4}$ on the "exposome" begins to acknowledge the advantages of external markers of exposure. I am confident that external marker measurements will continue play a fundamental role in prevention of contact or an intervention to eliminate contact with 
a toxicant or multiple toxicants in long-term or acute exposure situations. $^{2}$

To re-enforce the need Exposure Scientists and others to use both internal and external exposure measurements; incorporate model estimates to solve exposure science problems; and indicate that ecosystems are part of the dynamics of the field, a recent NRC Committee introduced a new term, Eco-exposome. ${ }^{5}$ The intent was not just to introduce another term to the field of exposure science, but to project a positive image for exposure science that better defines the large reach that it has in understanding and mitigating contacts with toxic materials that can result in an adverse outcome, human disease or otherwise. The Eco-exposome is defined as: the extension of exposure science from the point of contact between stressor and receptor inward into the organism and outward to the general environment, including the ecosphere. Thus, it includes the features of the exposome and at the same time reminds us that research in exposure science has many features that span the source-to-effects continuum first described by me and others since the late 1980s. The European Commission actually is doing the science correctly. They are funding a major study, with the lead at Imperial College, London, in which, to quote Nature (2012), "Subjects will carry smartphones equipped with sensors to measure exposures, and their blood will be analyzed to monitor molecular changes." ${ }^{\prime \prime}$ This approach is conceptually similar to the Eco-exposome as it employs two key components of exposure science, internal and external markers.

I strongly recommend that we turn our energies toward supplying the ideas and the resources necessary to mitigate current exposures and prevent new exposures harmful to both humans and the ecosystem. Embracing the major recommendations in our recent NRC report, Exposure Science in the 21st Century is a good starting point. ${ }^{5} \mathrm{I}$ am encouraged by the effort being made by EPA and other US federal agencies to begin to address its recommendations.

\section{CONFLICT OF INTEREST}

The author declares no conflict of interest.

Paul J. Lioy

Rutgers Environmental and Occupational Health Sciences Institute, Piscataway, NJ 08854, USA E-mail: plioy@eohsi.rutgers.edu

\section{REFERENCES}

1 Wild CP. Complementing the genome with an "exposome": the outstanding challenge of environmental exposure measurement in molecular epidemiology. Cancer Epidemiol Biomarkers Prev 2005; 14: 1847-1850.

2 Lioy PJ. Exposure science: a view of the past and milestones for the future. Environ Health Perspect 2010; 118: 1081-1090.

3 Lioy PJ, Rappaport SM. Exposure science and the exposome: an opportunity for coherence in the environmental health sciences. Environ Health Perspect 2011; 119: A466-A467.

4 Wild CP. The exposome: from concept to utility. Int J Epidemiol 2012; 41: 24-32.

5 NRC, 2012. Exposure Science in the 21st Century: A Vision and A Strategy. National Research Council: Washington, DChttp://www.nap.edu/catalog.php?record id $=13507$

6 Callaway E. Daily dose of toxins to be tracked. Nature 2012; 491: 647. 